\title{
Prematuros tardíos: un grupo de riesgo de morbilidad a corto y largo plazo
}

\author{
LUISA SCHONHAUT B. ${ }^{1}$, MARCELA PÉREZ R. ${ }^{2}$, JULIO ASTUDILLO D. ${ }^{3}$ \\ 1. Pediatra, Magister en Salud Pública. Facultad de Medicina Clínica Alemana - Universidad del Desarrollo. \\ 2. Pediatra, Neonatóloga. Facultad de Medicina Clínica Alemana - Universidad del Desarrollo. \\ 3. Ginecoobstetra. Facultad de Medicina Clínica Alemana - Universidad del Desarrollo.
}

\begin{abstract}
Late preterm infants: a population at risk in the short and long term

Preterm Birth has increased over the last decades in developed countries; the mayor contribution to this increase has been from "late preterm" births (LP). Recent publications show higher rates of mortality and morbidity for LP compared with full term infants, both in the short and long term; the risk of mortality and morbidity increases with decreasing gestational age, the lowest risk being at 39 weeks of gestation. Complications of LP birth are related to immaturity of the different organs and systems. During the neonatal period, the most frequent complications are Hyaline Membrane Disease, Transient Tachipnea, Hypoglycemia, Hyperbilirubinemia and feeding problems. In the long term, outcomes show that LP have an increased risk for developmental delay, academic and behavioral difficulties. While most complications are less frequent and less severe than extremely preterm infants, the large number of children that are born as LP represent a large population at risk. The purpose of this article is to review available evidence on mortality, morbidity and long term development in LP. This data may help obstetricians, neonatologists, pediatricians and public health workers to pay attention to this new population at risk.
\end{abstract}

(Key words: Late preterm, neonatal morbidity, psychomotor development).

Rev Chil Pediatr 2012; 83 (3): 217-223

\section{RESUMEN}

La prematurez ha aumentado a lo largo de las últimas décadas en países desarrollados, fundamentalmente en base al incremento de los Prematuros Tardíos (PT). Publicaciones recientes evidencian que los PT presentan tasas de mortalidad y morbilidad, tanto a corto como a largo plazo, superiores que los niños nacidos a término; esta probabilidad es inversa a la edad gestacional y, alcanza el nadir a las 39 semanas de gestación. En el periodo neonatal predominan las complicaciones relacionadas con la inmadurez de los distintos órganos y sistemas, como la Enfermedad de Membrana Hialina, Taquipnea Transitoria, Hipoglicemia, Hiperbilirrubinemia y los problemas de alimentación. A largo plazo, los PT tendrían mayor riesgo de presentar dificultades del desarrollo, aprendizaje y comportamiento. Si bien la mayoría de las complicaciones son menos frecuentes y

Correspondencia a:

Luisa Schonhaut Berman

Ischonhaut@alemana.cl 
menos severas que en prematuros extremos, debido al extenso número de niños que nacen bajo esta condición, constituyen una importante fracción poblacional con morbimortalidad atribuible a la prematuridad tardía. El objetivo del artículo es revisar la evidencia disponible respecto a la mortalidad, morbilidad y desarrollo de los PT y alertar a obstetras, neonatólogos, pediatras y salubristas sobre este nuevo grupo de riesgo.

(Palabras clave: Prematuros tardíos, morbilidad neonatal, desarrollo psicomotor).

Rev Chil Pediatr 2012; 83 (3): 217-223

\section{Introducción}

El año 2009, 12,2\% de los nacimientos en USA y $7,2 \%$ en nuestro país fueron prematuros, es decir, menores de 37 semanas de edad gestacional (EG); de estos, cerca del 70\% nacieron entre las 34 y $36^{6}$ semanas, grupo actualmente denominado prematuros tardíos (PT) $(\text { tabla } 1)^{1-5}$.

La prematurez ha aumentado a lo largo de las últimas décadas en países desarrollados, fundamentalmente en base al incremento de los $\mathrm{PT}^{2,6}$. A nivel nacional, mientras la tasa global de natalidad descendió entre los años 1994 y 2009, los nacimientos PT mantuvieron un aumento sostenido, equivalente al $41 \%$ el mismo período ${ }^{1}$ (figura 1).

Existen distintos factores que contribuyen al reciente ascenso de la prematuridad. Por un lado, el incremento de la edad materna se asocia a mayor riesgo obstétrico y por ende interrupciones prematuras de la gestación ${ }^{7}$. Por otro lado, los embarazos múltiples, ya sean espontáneos, frecuentes en madres de mayor edad, o inducidos por programas de reproducción asistida, también han ido en aumento, siendo responsables del 15 a 20\% de los nacimientos prematuros ${ }^{1,8}$ (figura 2).

Tabla 1. Proporción de nacidos vivos segú n EG, Chile 2009

\begin{tabular}{|cc|}
\hline Edad gestacional & (\%) \\
$39-42$ & 56,85 \\
$37-38$ & 35,95 \\
$34-36$ & 5,12 \\
$32-33$ & 0,94 \\
$28-31$ & 0,72 \\
$<28$ & 0,42 \\
\hline
\end{tabular}

Fuente DEIS 1 .
A estos factores se han sumado los avances en los métodos científicos y tecnológicos orientados a la vigilancia obstétrica, que permiten una detección precoz de problemas maternos, placentarios y fetales, apoyando la toma de decisiones frente a la necesidad de intervenir la gestación, con el propósito final de disminuir la mortalidad materno-fetal ${ }^{9,10}$.

Es probable que, debido a la convicción que a partir de las 34 semanas los prematuros son "cercanos a término" y por ende ya maduros para enfrentarse al ambiente extrauterino, el criterio obstétrico para decidir la interrupción de la gestación, sea menos estricto en esa etapa, que a menor $\mathrm{EG}^{11,12}$. De hecho, los recién nacidos mayores de 34 semanas, con buen peso y ausencia de patología inmediata al nacimiento, son considerados "libres de riesgo" y consecuentemente reciben los cuidados neonatales y pediátricos habituales.

Esta percepción de falta de vulnerabilidad tiene su base histórica. Hace 75 años, se consideraba que todo niño que nacía con peso inferior a 2500 gr era prematuro y requería de un "cuidado especial", siendo la sobrevida de los nacidos con peso inferior a 1000 gr prácticamente anecdótica ${ }^{13}$. Producto del desarrollo de la neonatología fue mejorando la sobrevida de prematuros cada vez más pequeños, concentrando la atención y recursos de los sistemas de salud. A nivel mundial, los programas de seguimiento están enfocados a los prematuros menores de 32 semanas de gestación y/o 1500 gr, reportando resultados cada vez más alentadores ${ }^{14}$.

La renovada atención por los prematuros "cercanos al termino" data del año 2005, en que se generó un consenso mundial respecto a considerarlos como un grupo de riesgo biológico, basado en la evidencia que son fisiológicamente inmaduros, $\mathrm{y}$, por ende, tienen eleva- 


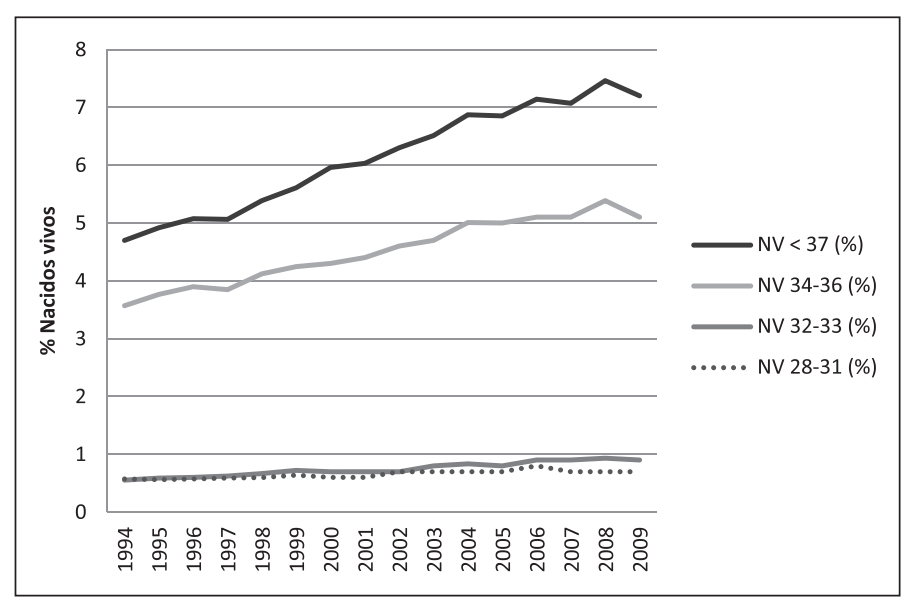

Figura 1. Evolución de la prematurez según edad gestacional al nacer. Chile 1994-2009. NV = Nacidos vivos. Fuente: elaboración propia a partir de información del DEIS ${ }^{1}$.

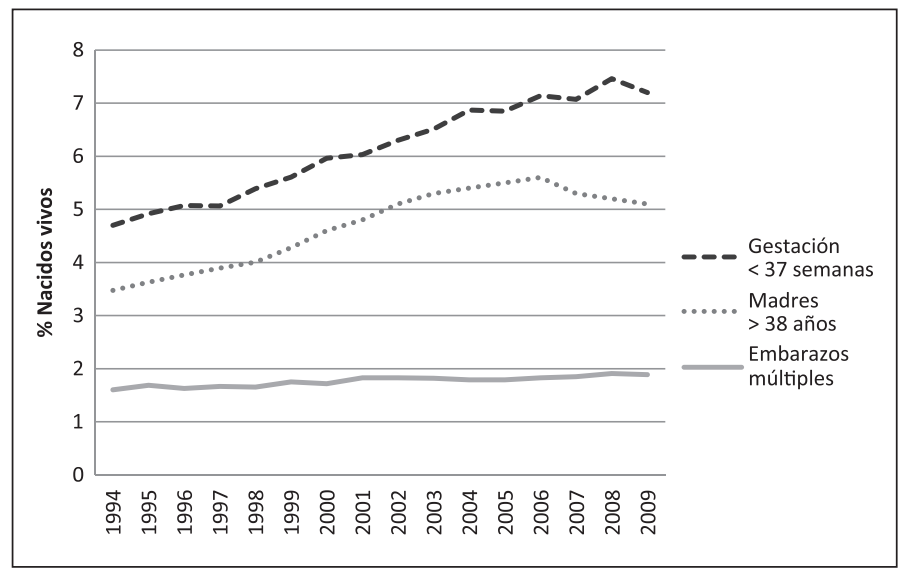

Figura 2. Evolución de factores de alto riesgo obstétrico, como embarazos múltiples y gestantes mayores de 38 años y prematuridad. Chile 1994-2009. Fuente: elaboración propia a partir de información del DEIS ${ }^{1}$.

do riesgo de mortalidad y morbilidad tanto a corto como a largo plazo ${ }^{15-17}$. A partir de entonces, se acordó calificarlos como "Prematuros Tardíos" para enfatizar la vulnerabilidad inherente a la condición de prematuridad ${ }^{4,5}$.

Si bien las complicaciones de los PT son francamente de menor severidad y menor frecuencia que los prematuros extremos, su relevancia para la Salud Pública radica en el extenso número de niños que nacen con este factor riesgo, lo que se traduce en una importante fracción poblacional con morbimortalidad atribuible a esta condición ${ }^{18}$. Por este motivo nos pareció relevante revisar la evidencia disponible respecto a la mortalidad, morbilidad y desarrollo de los PT y alertar a obstetras, neonatólogos, pediatras y salubristas sobre este nuevo grupo de riesgo.

\section{Morbi-mortalidad de los prematuros tardíos}

Numerosas publicaciones constatan que los PT tienen una elevada morbilidad y mortalidad comparado con los RNT, tanto en el periodo neonatal como a más largo plazo. Una revisión sistemática reciente pone en manifiesto que los riesgos relativos de mortalidad neonatal precoz, neonatal tardía y postneonatal son del orden de 5, 6 y 4 veces mayores en los $\mathrm{PT}^{17,19}$. En nuestro país, la mortalidad neonatal precoz el año 2009 fue de 10,3 por mil nacidos vivos en los PT y 0,55 por mil en los RNT 6 .

En cuanto a la morbilidad, se ha reportado que un tercio de los prematuros de 34 semanas de EG podrían hospitalizarse en el periodo neonatal, comparado con $0,5 \%$ de los $\mathrm{RNT}^{19}$. Este riesgo, que se mantendría elevado durante el resto de la infancia, guarda relación inversa con la EG, y alcanza el nadir a las 39 semanas de gestación ${ }^{12,20,21}$. Esta relación sugiere un efecto "dosisrespuesta" de la prematuridad y nos plantea que los niños nacidos de término temprano también sumarían mayor riesgo neonatal inmediato y de morbilidad posterior ${ }^{18,22}$.

Al revisar las principales causas de hospitalización neonatal de los PT, se infiere que podría ser la inmadurez al momento del parto, la causante fundamental de los problemas médicos, en su mayoría atribuibles a las dificultades de "adaptación" a la vida extrauterina. Es así como entre las complicaciones respiratorias son frecuentes la Enfermedad de Membrana Hialina, la Taquipnea Transitoria y las Apneas; entre los trastornos metabólicos prevalecen la Hipoglicemia, Hiperbilirrubinemia, e Hipocalcemia. Los problemas de 
Tabla 2. Morbilidad neonatal precoz de los prematuros tardíos

\begin{tabular}{|lcccc|}
\hline & $\begin{array}{c}\text { PT } \\
(\%)\end{array}$ & $\begin{array}{c}\text { RNT } \\
(\mathbf{\%})\end{array}$ & RR & (IC 95\%) \\
\hline Membrana Hialina & 5,30 & 0,39 & 17,3 & $(9,8-30,6)$ \\
\hline Taquipnea transitoria & 3,50 & 0,45 & 7,5 & $(5,0-11,2)$ \\
\hline Hipertensión Pulmonar & 0,40 & 0,08 & 4,9 & $(3,8-6,3)$ \\
\hline Apneas & 0,87 & 0,05 & 15,7 & $(11,8-20,9)$ \\
\hline Neumotórax & 0,81 & 0,17 & 3,4 & $(1,8-6,4)$ \\
\hline Hiperbilirrubinemia & 4,70 & 1,30 & 5 & $(1,7-14,6)$ \\
\hline Hipoglicemia & 7,10 & 0,70 & 7,4 & $(3,0-18,1)$ \\
\hline Hipotermia & 1,50 & 0,08 & 10,8 & $(4,6-25)$ \\
\hline Enterocolitis Nerotizante & 0,11 & 0,01 & 7,5 & $(3,3-17,3)$ \\
\hline Sepsis sospechada & 19,70 & 11,80 & 4,2 & $(1,7-10,7)$ \\
\hline Hemorragia intracraneana & 0,40 & 0,09 & 4,9 & $(2,1-11,7)$ \\
\hline Problemas de alimentación & 34,00 & 6,70 & 6,5 & $(2,5-17)$ \\
\hline
\end{tabular}

Adaptado de Teune M. A Sistematic Review of severe morbility in infants born late preterm ${ }^{17}$.

alimentación dados por la inmadurez de la coordinación de succión-deglución-respiración, también son importantes causas de hospitalización precoz, prolongación de la estadía hospitalaria y reingresos posteriores, debido a sus complicaciones como la deshidratación e hiperbilirrubinemia ${ }^{17}$ (tabla 2).

Otros problemas médicos menos frecuentes, pero de gran relevancia por su severidad son el Ductus arterioso persistente, la Enterocolitis necrotizante y la Hemorragia intraventricular, esta última si bien poco probable en grados mayores, se concentraría en el grupo de PT con restricción del crecimiento intrauterino ${ }^{23}$.

Durante el primer año de vida también tienen mayor incidencia de hospitalización, predominando las infecciones respiratorias y los problemas gastrointestinales en este periodo ${ }^{21}$. A lo largo de la infancia, se evidencia mayor prevalencia de morbilidad respiratoria y el compromiso de la función pulmonar, especialmente en aquellos PT que presentaron problemas respiratorios en periodo neonatal inmediato ${ }^{18,24}$.

En vista de la evidencia actual, no podemos atribuir a la prematuridad todo el riesgo de morbilidad de los PT, siendo importante considerar en esta ecuación las causas que condicionaron el nacimiento de pretérmino y también la vía del parto ${ }^{25}$. Hay partos prematuros espontáneos y otros tienen indicación médica, ya sea por problemas fetales, maternos o placentarios. Se ha postulado que un embarazo patológico afecta el desarrollo fetal y la adaptación posterior, tanto a corto como a largo plazo ${ }^{12}$. La hipótesis más conocida al respecto, se refiere a la reprogramación fetal frente a la restricción de crecimiento intrauterino y su asociación con enfermedades crónicas no transmisibles en la vida adulta ${ }^{26}$.

Otro aspecto que plantea nuevas controversias y desafíos es el uso de corticoides antenatales, cuyo beneficio se ha demostrado en los prematuros menores de 34 semanas de EG y en el grupo de PT nacidos por cesárea sin trabajo de parto ${ }^{27}$. Es probable que durante el trabajo de parto ocurran procesos que aún no se conocen a cabalidad, que induzcan la madurez de los distintos órganos y sistemas y nos preparen para la adaptación al mundo exterior ${ }^{25}$.

Finalmente, es importante considerar que la hospitalización y complicaciones precoces que presentan los PT tienen un alto costo médico, que llega a superar hasta diez veces los costos asociados a los partos de término ${ }^{21,28}$. También es importante analizar los costos emocionales del binomio madre-hijo, cuyo efecto a largo plazo no ha sido aún bien establecido ${ }^{29}$.

\section{Morbilidad a largo plazo: dificultades en el desarrollo y el comportamiento}

En los últimos 2 a 3 años han ido in aumento las publicaciones acerca del desempeño psicomotor y académico de los PT. La mayoría de los estudios señala que los prematuros no extremos presentan prevalencias de déficit del desarrollo y dificultades de aprendizaje superiores a los RNT, con mayor compromiso en el área cognitiva $^{30-35}$. Estudios recientes nos alertan además sobre una elevada prevalencia de problemas en la esfera de la salud mental, como trastornos emocionales, problemas del comportamiento y Síndrome de Déficit Atencional, aspectos que podrían contribuir al menor rendimiento académico ${ }^{36-39}$. Parecería que el riesgo es inversamente proporcional a la EG, alcanzando un nadir 
a las 39 semanas, al igual que se ha reportado en relación a la morbilidad general ${ }^{31,40-42}$.

A pesar que la evidencia es cada vez más contundente, los estudios son relativamente nuevos, la mayoría corresponde a cohortes poblacionales retrospectivas, y carecen de uniformidad en la edad de evaluación, en las escalas de medición utilizadas y en el análisis de las variables confundentes, quedando varias controversias por resolver. Por ejemplo, la falta de acuerdo en relación a la corrección de la EG de los PT, podría conducir a conclusiones contradictorias; se ha demostrado que al corregir esta variable en lactantes y preescolares pequeños las desventajas se estrechan ${ }^{34,43}$.

Otro aspecto relevante, discutido en las últimas publicaciones, se relaciona con el impacto de la morbilidad neonatal en el desempeño posterior; se ha reportado que el rendimiento de PT de mayor edad EG y libres de morbilidad perinatal, podría ser similares a los $\mathrm{RNT}^{44,45}$ por el contrario, los PT que estuvieron hospitalizados en el periodo neonatal tendrían indicación de programas de apoyo al desarrollo similar a los prematuros extremos ${ }^{46}$.

Las diferencias en el rendimiento de los PT en comparación a los RNT tienen su base fisiológica, habiéndose demostrado que el cerebro completa su crecimiento y desarrollo después de las 38 semanas de gestación. Se plantea que podría ser la exposición de un cerebro aún inmaduro a las noxas del ambiente extrauterino, como por ejemplo hipoxia, hipoglicemia, hiperbilirubinemia, lo que podría desencadenar una menor organización neuronal, con las consecuencias mencionadas a nivel de desarrollo, aprendizaje y comportamiento ${ }^{47-49}$.

Sin duda el desarrollo y la salud mental son multifactoriales, incidiendo en ellos otros aspectos a lo largo de la vida, los que no han sido exahustivamente analizados en los estudios publicados. Para aclarar las interrogantes planteadas es fundamental contar con estudios prospectivos a largo plazo, que nos permitan conocer si en esta población de prematuros hay un catch-up cognitivo en etapas más tardías, o bien las diferencias persisten a lo largo de la vida, manifestándose como problemas en la esfera psiquiátrica y social en la adultez, como postulan algunos autores ${ }^{50-52}$.

\section{Reflexiones y recomendaciones finales}

Los PT nos plantean una serie de desafíos a nivel obstétrico, neonatal y pediátrico, que hemos intentado resumir a lo largo del artículo. Si bien está demostrado que concentran mayor riesgo de morbilidad tanto a corto, como mediano y largo plazo, investigaciones adicionales hacen falta para determinar cuál es el peso de la prematuridad en sí, y cuánto aportan otros factores, como la morbilidad materna u ovular que llevó al parto prematuro, la vía del parto e incluso las intervenciones perinatales.

A la luz de la evidencia actual, a nivel nacional se deberían desarrollar estrategias de cuidado de los PT, asumiendo el desafío de mejorar el pronóstico de cerca del 5\% de los niños que nacen hoy en nuestro país. Se propone implementar estándares de cuidado y prevención del parto prematuro tardío, diagnóstico y manejo oportuno de las complicaciones neonatales y programas de seguimiento y estimulación durante el resto de la infancia.

\section{Referencias}

1.- Departamento de Estadísticas e Información de Salud (DEIS), Ministerio de Salud. En htpp://www.deis.cl. (visitado el 03/06/12).

2.- Michael J. Davidoff, Todd Dias, Karla Damus, et al: Changes in the Gestational Age Distribution among U.S. Singleton Births: Impact on Rates of Late Preterm Birth, 1992 to 2002. Semin Perinatol 30:8-15. 2006.

3.- Martin JA, Hamilton BE, Ventura SJ, et al: Births: Final data for 2009. National vital statistics reports 2011; 60(1). Available from: http://www.cdc.gov/nchs/data/ nvsr/nvsr60/nvsr60_01.pdf

4.- Raju TNK, Higgins RD, Stark AR, Leveno KJ: Optimizing care and outcome for late-preterm (near-term) infants: a summary of the workshop sponsored by the National Institute of Child Health and Human Development. Pediatrics 2006; 118 (3): 1207-14.

5.- Engle WA: A Recommendation for the Definition of "Late Preterm" (Near-Term) and the Birth Weight-Gestational Age Classification System. Semin Perinatol 2006; 30: 2-7.

6.- González R, Nien KHK, Vera C, et al: i Existe un aumento de los nacimientos en Chile en el período 2000-2009? Análisis de los principales indicadores 
materno-infantiles de la década. Rev Chil Obstet 2011; 76 (6): 404-11.

7.- Donoso E, Villarroel L: Edad materna avanzada y riesgo reproductivo. Rev Med Chile 2003; 131 (1): 55-9.

8.- Lee YM, Cleary-Goldman J, D'Alton ME: Multiple gestations and late preterm (near-term) deliveries. Semin Perinatol 2006; 30: 103.

9.- Spong CY, Mercer BM, D'alton $M$, et al: Timing of indicated late-preterm and early-term birth. Obstet Gynecol 2011; 118: 323

10.- Engle WA, Kominiarek $M A$ : Late preterm infants, early term infants, and timing of elective deliveries. Clin Perinatol 2008; 35: 325.

11.- Wang ML, Dorer DJ, Fleming MP, Catlin EA: Clinical outcomes of near-term infants. Pediatrics 2004; 114: 372.

12.- Shapiro-Mendoza C, Tomashek K, Kotelchuck M, et al: Effect of Late-Preterm Birth and Maternal Medical Conditions on Newborn Morbidity Risk. Pediatrics 2008; 121: e223-32.

13.- Schonhaut L, Pérez M: Estudio de 258 Prematuros en el Primer Año de Vida. Rev Chil Pediatr 2010; 81 (3): 253-60.

14.- American Academy of Pediatrics: Committee on Fetus and Newborn. Hospital discharge of the high-risk neonate-proposed guidelines. Pediatrics 1998; 102: 411-7.

15.- Wang ML, Dorer DJ, Fleming MP, Catlin EA: Clinical outcomes of near-term infants. Pediatrics 2004; 114: 372.

16.- Engle WA, Tomashek KM, Wallman C: American Academy of Pediatrics, Committee on Fetus and Newborn. "Late-preterm" infants: a population at risk. Pediatrics 2007; 120: 1390-401.

17.- Teune MJ, Bakhuizen S, Gyamfi Bannerman C, et al: A systematic review of severe morbidity in infants born late preterm. American journal of obstetrics and gynecology 2011; 205 (4): 374.

18.- Boyle EM, Poulsen G, Field DJ, et al: Effects of gestational age at birth on health outcomes at 3 and 5 years of age: population based cohort study. BMJ 2012; 344 : e896-e896.

19.- McIntire D, Leveno K: Neonatal Mortality and Morbidity Rates in Late Preterm Births Compared With Births at Term. Sepsis 2008; 111 (1): 35-41.

20.- Bastek JA, Sammel MD, Paré E, et al: Adverse neonatal outcomes: examining the risks between preterm, late preterm, and term infants. Am J Obstet Gynecol 2008; 199: 367.e1-8.

21.- McLaurin KK, Hall CB, Jackson EA, Owens OV, Mahadevia PJ: Persistence of morbidity and cost differences between late-preterm and term infants during the first year of life. Pediatrics 2009; 123 (2): 653-9.

22.- Armadans M, Ossorio MF, Pedicone C, Durán P, Ferrero F: Morbilidad en Recién Nacidos de Término en Relación a su Edad Gestacional. Rev Chil Pediatr 2010; 81 (5): 402-8.

23.- Ortigosa C, Bittar E, Zugaib M: Neonatal Outcome of Late Preterm Birth Associated or Not with Intrauterine Growth Restriction. Obstetrics and Gynecology International 2010; article ID 231842. En: http://www. hindawi.com/journals/ogi/2010/231842/cta/ (visitado el 03/06/12).

24.- Colin A, McEvoy $C$, Castile R: Respiratory morbidity and lung function in preterm infants of 32 to 36 weeks' gestational age. Pediatrics 2010; 126 (1): 115-28.

25.- Ramachandrappa A, Jain L: Elective Cesarean Section: Its Impact on Neonatal Respiratory Outcome Review Article. Clin Perinatol 2008; 35 (2): 373-93.

26.- Barker DJ: The developmental origins of chronic adult disease. Acta Paediatr Suppl 2004; 2003: 26-33.

27.- Feitosa AM, Coutinho IC, Ramos MM: Effectiveness of antenatal corticosteroids in reducing respiratory disorders in late preterm infants: randomised clinical trial. BMJ 2011; 342: d1696.

28.- Stavros P, Kahn K: Economic costs associated with moderate and late preterm birth: Primary and secondary evidence. Semin Fetal Neonatal Med 2012; 12 (3): 1708.

29.- Miles MS, Funk SG, Kasper MA: The stress response of mothers and fathers of preterm infants. Res Nurs Health 1992;15: 261-9.

30.- Chyi LJ, Lee HC, Hintz SR, Gould JB, Sutcliffe TL: School outcome of late preterm infants: special needs and challenges for infants born at 32 to 36 weeks gestation. J Pediatr 2008; 153: 25-31.

31.- Morse SB, Zheng H, Tang Y, Roth J: Early School-Age Outcomes of Late Preterm Infants. Pediatrics 2009; 123 : e622-9.

32.- Van Baar AL, Vermaas J, Knots E, De Kleine MJ, Soons $P$ : Functioning at School Age of Moderately Preterm Children Born at 32 to 36 Weeks' Gestational Age. Pediatrics 2009; 124: 251-7.

33.- Petrini JR, Dias T, McCormick MC, et al: Increased risk of adverse neurological development for late preterm infants. J Pediat 2009; 154 (2): 169-76.

34.- Schonhaut L, Pérez M, Schonstedt M, et al: Prematuros moderados y tardíos, un grupo de riesgo de menor desarrollo cognitivo en los primeros años de vida. Rev Chil Pediatr 2012; 83 (4). En prensa.

35.- Peacock PJ, Henderson J, Odd D, Emond A: Early 
school attainment in late-preterm infants. Arch Dis Child 2012; 97 (2): 118-20

36.- Huddy CL, Johnson A, Hope PL: Educational and behavioural problems in babies of 32-35 weeks gestation. Arch Dis Child Fetal Neonatal Ed 2001; 85: F23-8.

37.- Linnet KM, Wisborg K, Agerbo E, Secher NJ, Thomsen $P H$, Henriksen TB: Gestational age, birth weight, and the risk of hyperkinetic disorder. Arch Dis Child 2006; 91 (8): 655-60.

38.- Talge NM, Holzman C, Wang J, et al: Late-preterm birth and its association with cognitive and socioemotional outcomes at 6 years of age. Pediatrics 2010; 126 (6): 1124-31.

39.- Potijk MR, de Winter AF, Bos AF, Kerstjens JM, Reijneveld $S A$ : Higher rates of behavioural and emotional problems at preschool age in children born moderately preterm. Arch Dis Child 2012; 97 (2): 112-7.

40.- Mathiasen R, Hansen B, Nybo Andersen AM, Forman J, Greisen G: Gestational Age and Basic School Achievements: A National Follow-up Study in Denmark. Pediatrics 2010; 126: e1553-61.

41.- Lipkind HS, Slopen ME, Pfeiffer MR, McVeigh KH: School-age outcomes of late preterm infants in New York City. American Journal of Obstetrics and Gynecology 2012; 206 (3): 222.e1-6.

42.- Yang S, Platt RW, Kramer MS: Variation in child cognitive ability by week of gestation among healthy term births. Am J Epidemiol 2010; 171 (4): 399-406.

43.- Romeo DM, Di Stefano A, Conversano M, et al: Neurodevelopmental outcome at 12 and 18 months in late preterm infants. Eur J Paediatr Neurol 2010; 14 (6): 503-7.

44.- Gurka M, Locasale-Crouch J, Blackman J: Long-term
Cognition, Achievement, Socioemotional, and Behavioral Development of Healthy Late-Preterm Infants. Arch Pediatr Adolesc Med 2010; 164 (6): 525-32.

45.- Romeo DM, Guzzardi S, Ricci D, et al: Longitudinal cognitive assessment in healthy late preterm infants. European journal of paediatric neurology: EJPN: official Journal of the European Paediatric Neurology Society. 2012; 16 (3): 243-7.

46.- Kalia JL, Visintainer P, Brumberg HL, Pici M, Kase J: Comparison of enrollment in interventional therapies between late-preterm and very preterm infants at 12 months' corrected age. Pediatrics 2009; 123 (3): 804-9.

47.- Martell M, Burgueño M, Arbón G, et al: Asociación entre morbilidad neonatal y desarrollo en pretérminos a la edad escolar. Arch Pediatr Urug 2007; 78 (2): 99-109.

48.- Nomura Y, Halperin J, Newcorn J, et al: The Risk for Impaired Learning-related Abilities in Childhood and Educational Attainment Among Adults Born Near-term . Journal of Pediatric Psychology 2009; 34 (4): 406-18.

49.- Baron IS, Erickson K, Ahronovich M, Baker R, Litman $F$ : Cognitive deficit in preschoolers born late-preterm. Early Human Development 2011; 87: 115-9.

50.- Moster D, Lie RT, Markestad T: Long-term medical and social consequences of preterm birth. N Engl J Med 2008; 359: 262.

51.- Lindström K, Winbladh B, Haglund B, Hjern A: Preterm Infants as Young Adults: A Swedish National Cohort Study. Pediatrics 2007; 120 (1): 70-7.

52.- Lindström K, Lindblad F, Hjern A: Psychiatric morbidity in adolescents and young adults born preterm: a Swedish national cohort study. Pediatrics 2009; 123 (1): e47-e53. 